JOURNAL OF

FUNCTION SPACES AND APPLICATIONS

Volume 1, Number 1 (2003), 1-15
(C) 2003, Scientific Horizon http://www.jfsa.net

\title{
Hardy operator with variable limits on monotone functions ${ }^{1}$
}

\author{
Vladimir D. Stepanov and Elena P. Ushakova
}

(Communicated by Lars-Erik Persson)

2000 Mathematics Subject Classification. 26D15.

Keywords and phrases. Weighted integral inequalities for monotone functions.

\footnotetext{
Abstract. We characterize weighted $L^{p}-L^{q}$ inequalities with the Hardy operator of the form $\mathbb{H} f(x)=\int_{a(x)}^{b(x)} f(y) u(y) d y$ with a non-negative weight function $u$, restricted to the cone of monotone functions on the semiaxis. The proof is based on the Sawyer criterion and the boundedness of generalized Hardy operator with variable limits.
}

\section{Introduction}

We study the Hardy operator

$$
\mathbb{H} f(x)=\int_{a(x)}^{b(x)} f(y) u(y) d y
$$

with a non-negative weight function $u$, restricted to the cone of non-increasing or non-decreasing non-negative functions on the semiaxis $\mathbb{R}^{+}:=[0, \infty)$. The border functions $a(x)$ and $b(x)$ are supposed to be continuous and strictly increasing on $\mathbb{R}^{+}$and as a model case may be taken such that

$$
0=a(0)=b(0)<a(\infty)=b(\infty)=\infty .
$$

\footnotetext{
${ }^{1}$ The research work was financially supported by the Russian Foundation for Basic Research (project 03-01-00017) and by the Far Eastern Branch of RAS.
} 
The equalities in (2) are not important as well as continuity of the border functions and assumed for simplicity. Also for simplicity we assume that $a(x) \neq b(x)$ everywhere on $\mathbb{R}^{+}$except a countable subset.

The operator (1) with $u=1$ was studied in [1], but the only sufficient condition for weighted $L^{p}-L^{q}$ boundedness on monotone functions was found there. A natural way to solve this problem is to apply the Sawyer criterion [2]. However, it leads to the additional problem for weighted $L^{p}-$ $L^{q}$ characterization of generalized Hardy operators. This was done in [3] for the case $a(x)<b(x), x>0$ (see also, [4], [5]). Using the results of [3] and the block-diagonal structure of (1) we characterize the operator $\mathbb{H}$ on monotone functions.

The paper is organized as follows. Section 2 contains the weighted $L^{p}-$ $L^{q}$ characterization of generalized Hardy operators. In particular, for the operator $\mathbb{H}$, the "fairway" form of criteria are given. In Section 3 we remind the Sawyer criterion and formulate its dual form, more convenient for our purpose. The main result is given in Section 4.

Throughout of the paper products of the form $0 \cdot \infty$ are taken to be equal to 0 . Relations $A \ll B$ mean $A \leqslant c B$ with some constants $c$ depending only on parameters of summations and, possibly, on the constants of equivalence in the inequalities of the type (8). We write $A \approx B$ instead of $A \ll B \ll A$ or $A=c B . \mathbb{Z}$ denotes the set of all integers and $\chi_{E}$ stands for a characteristic function (indicator) of a subset $E$. The union of disjoint sets we denote by $\sqcup$ and $\sqcup$. Also we make use of marks : $=$ and $=$ : for introducing new quantities as well as the symbol $\square$ for the end of proof.

\section{Generalized Hardy operator}

Let

$$
K f(x)=\int_{a(x)}^{b(x)} k(y, x) f(y) d y, \quad x>0,
$$

with a measurable kernel $k(x, y) \geqslant 0$ and strictly increasing functions $a(x)$ and $b(x)$, satisfying (2) such that $a(x) \neq b(x)$ for $0<x<\infty$ except $x \in X$, where

$$
X:=\left\{x_{k}\right\}, \quad k \in \Sigma \subset \mathbb{Z} \quad \text { and } \quad a\left(x_{k}\right)=b\left(x_{k}\right) .
$$

Denote

$$
\begin{gathered}
(0, \infty)=: I \sqcup J, \\
I=: \bigsqcup_{i \in \Sigma_{1}} I_{i}, \quad J=: \bigsqcup_{j \in \Sigma_{2}} J_{j}, \quad \Sigma=\Sigma_{1} \sqcup \Sigma_{2}, \\
I_{i}=\left(t_{i}, t_{i+1}\right), \quad J_{j}=\left(s_{j}, s_{j+1}\right),
\end{gathered}
$$

where $\left\{t_{i}\right\}_{i \in \Sigma_{1}} \subset\left\{x_{k}\right\}_{k \in \Sigma},\left\{s_{j}\right\}_{j \in \Sigma_{2}} \subset\left\{x_{k}\right\}_{k \in \Sigma}$ such that

$$
x \in I_{i} \quad \Leftrightarrow \quad a(x)<b(x),
$$




$$
x \in J_{j} \quad \Leftrightarrow \quad b(x)<a(x) .
$$

Suppose that there exists $D \geqslant 1$ such that for all $i \in \Sigma_{1}$ and $j \in \Sigma_{2}$ the condition

$$
D^{-1} k(y, x) \leqslant k(y, z)+k(a(z), x) \leqslant D k(y, x),
$$

holds, if

and, similarly,

$$
\left\{\begin{array}{l}
t_{i} \leqslant x \leqslant z \leqslant t_{i+1} \\
a(z) \leqslant y \leqslant b(x)
\end{array}\right.
$$

$$
D^{-1} k(y, x) \leqslant k(y, z)+k(b(z), x) \leqslant D k(y, x),
$$

when

$$
\left\{\begin{array}{l}
s_{j} \leqslant x \leqslant z \leqslant s_{j+1} \\
b(z) \leqslant y \leqslant a(x)
\end{array}\right.
$$

We call a linear operator $T: \mathcal{L}(U) \rightarrow \mathcal{M}(V)$ block-diagonal, if $U=$ $\bigsqcup_{k} U_{k}, V=\bigsqcup_{k} V_{k}$ and $T=\sum_{k} T_{k}$, where $T_{k}: \mathcal{L}\left(U_{k}\right) \rightarrow \mathcal{M}\left(V_{k}\right)$ and $T_{k} f=$ $\chi_{V_{k}} T\left(\chi_{U_{k}} f\right)$.

The following Lemma is frequently used. For completeness we provide the proof.

Lemma 1. Let $U=\bigsqcup_{k} U_{k}, V=\bigsqcup_{k} V_{k}$ and $T=\sum_{k} T_{k}$ is a blockdiagonal operator, $T_{k}: L^{p}\left(U_{k}\right) \rightarrow L^{q}\left(V_{k}\right)$. Then

$$
\|T\|_{L^{p}(U) \rightarrow L^{q}(V)}=\sup _{k}\left\|T_{k}\right\|_{L^{p}\left(U_{k}\right) \rightarrow L^{q}\left(V_{k}\right)}, \quad 0<p \leqslant q<\infty
$$

and

(11)

$\|T\|_{L^{p}(U) \rightarrow L^{q}(V)}=\left(\sum_{k}\left\|T_{k}\right\|_{L^{p}\left(U_{k}\right) \rightarrow L^{q}\left(V_{k}\right)}^{r}\right)^{1 / r}, 0<q<p<\infty, \frac{1}{r}=\frac{1}{q}-\frac{1}{p}$.

Proof. Let $0<p \leqslant q<\infty$. Since $\|T f\|_{L^{q}(V)} \geqslant\left\|T_{k} f\right\|_{L^{q}\left(V_{k}\right)}$ for all $k$, the lower estimate in (10) is trivial. For the upper bound, applying Jensen inequality, we write

$$
\begin{aligned}
\|T f\|_{L^{q}(V)}^{q} & =\sum_{k}\left\|T_{k} f\right\|_{L^{q}\left(V_{k}\right)}^{q} \\
& \leqslant\left(\sup _{k}\left\|T_{k}\right\|_{L^{p}\left(U_{k}\right) \rightarrow L^{q}\left(V_{k}\right)}\right)^{q} \sum_{k}\left\|f \chi_{k}\right\|_{L^{p}\left(U_{k}\right)}^{q} \\
& \leqslant\left(\sup _{k}\left\|T_{k}\right\|_{L^{p}\left(U_{k}\right) \rightarrow L^{q}\left(V_{k}\right)}\right)^{q}\|f\|_{L^{p}(U)}^{q} .
\end{aligned}
$$


Let $0<q<p<\infty$. The upper bound in (11) follows similar to (12) by application of Hölder's inequality. For the lower bound let $0<\lambda<1$ be given and the functions $f_{k} \in L^{p}\left(U_{k}\right)$ are such that for all $k$

$$
\begin{gathered}
\left\|f_{k}\right\|_{L^{p}\left(U_{k}\right)}=\left\|T_{k}\right\|_{L^{p}\left(U_{k}\right) \rightarrow L^{q}\left(V_{k}\right)}^{r / p}, \\
\lambda\left\|T_{k}\right\|_{L^{p}\left(U_{k}\right) \rightarrow L^{q}\left(V_{k}\right)}\left\|f_{k}\right\|_{L^{p}\left(U_{k}\right)} \leqslant\left\|T_{k} f_{k}\right\|_{L^{p}\left(U_{k}\right)} .
\end{gathered}
$$

The inequality here follows from the definition of the norm of a linear operator and then we can change the function $f_{k}$, if necessary, by multiplying on a constant for the equality is satisfied. If we put $f=\sum_{k} \chi_{U_{k}} f_{k}$, then

$$
\begin{aligned}
\lambda^{q} \sum_{k}\left\|T_{k}\right\|_{L^{p}\left(U_{k}\right) \rightarrow L^{q}\left(V_{k}\right)}^{r} & =\lambda^{q} \sum_{k}\left(\left\|T_{k}\right\|_{L^{p}\left(U_{k}\right) \rightarrow L^{q}\left(V_{k}\right)}\left\|f_{k}\right\|_{L^{p}\left(U_{k}\right)}\right)^{q} \\
& \leqslant \sum_{k}\left\|T_{k} f_{k}\right\|_{L^{p}\left(U_{k}\right)}^{q} \\
& =\|T f\|_{L^{q}(V)}^{q} \\
& \leqslant\|T\|_{L^{p}(U) \rightarrow L^{q}(V)}^{q}\|f\|_{L^{p}(U)}^{q} \\
& =\|T\|_{L^{p}(U) \rightarrow L^{q}(V)}^{q}\left(\sum_{k}\left\|T_{k}\right\|_{L^{p}\left(U_{k}\right) \rightarrow L^{q}\left(V_{k}\right)}^{r}\right)^{q / p}
\end{aligned}
$$

and the lower bound in (11) follows by tending $\lambda \rightarrow 1$.

Remark. Observe, that if $0<p<1 \leqslant q$ and $\|T\|<\infty$, then $\|T\|=0$ ([6], $\S 1.47)$ and, if $0<q<\infty, 0<p<1,\|T\|<\infty$ and $T$ is an integral operator, then $\|T\|=0([[7]$, Theorem 2).

We consider the inequality

$$
\left(\int_{0}^{\infty}|K f(x)|^{q} w^{q}(x) d x\right)^{1 / q} \leqslant C\left(\int_{0}^{\infty}|f(x)|^{p} v^{p}(x) d x\right)^{1 / p},
$$

where $w(x) \geqslant 0$ and $v(x) \geqslant 0$ are locally integrable functions (weights) and $K$ is given by (3) with the border functions $a(x)$ and $b(x)$ satisfying (2), (4-7) and the kernel $k(y, x) \geqslant 0$ satisfying (8) and (9).

Denote

$$
L_{v}^{p}:=\left\{f:\|f v\|_{p}:=\left(\int_{0}^{\infty}|f(x)|^{p} v^{p}(x) d x\right)^{1 / p}<\infty\right\}
$$

and let $\|K\|_{X \rightarrow Y}$ stand for the norm of $K: X \rightarrow Y$.

Obviously, under the above assumptions on $a(x)$ and $b(x) K$ has a blockdiagonal structure and Lemma 1 gives

$$
\|K\|_{L_{v}^{p} \rightarrow L_{w}^{q}}=\sup _{k \in \Sigma} \mathbb{A}_{k}, \quad 1<p \leqslant q<\infty
$$




$$
\|K\|_{L_{v}^{p} \rightarrow L_{w}^{q}}=\left(\sum_{k \in \Sigma} \mathbb{A}_{k}^{r}\right)^{1 / r}, \quad 1<q<p<\infty,
$$

where

$$
\mathbb{A}_{k}=\|K\|_{L_{v}^{p}\left(a\left(U_{k}\right)\right) \rightarrow L_{w}^{q}\left(U_{k}\right)},
$$

$U_{k}$ runs the intervals $I_{i}$ and $J_{j}$, and $a(U)=\left(a\left(u_{1}\right), a\left(u_{2}\right)\right)$ if $U=\left(u_{1}, u_{2}\right) \subset$ $\mathbb{R}^{+}$.

The norms (16) we estimate with the help of [3] by the following scheme.

We have two types of the intervals $U_{k}$, namely $I_{i}$ (see (6)) and $J_{j}$ (see (7)). Since on each of $I_{i}$ the operator $K$ with the kernel $k(y, x) \in(8)$ satisfies the conditions of Theorem 2 [3], then

$$
\mathbb{A}_{i} \approx \begin{cases}A_{i}=A_{0}^{(i)}+A_{1}^{(i)}, & 1<p \leqslant q<\infty, \\ B_{i}=\left[\sum_{k \in \mathbb{Z}_{i}}\left(B_{k, 1}^{(i)}+B_{k, 2}^{(i)}+B_{k, 3}^{(i)}+B_{k, 4}^{(i)}\right)^{r}\right]^{1 / r}, & 1<q<p<\infty,\end{cases}
$$

where

$$
\begin{gathered}
A_{0}^{(i)}=\sup _{s \in I_{i}} \sup _{s \leqslant t \leqslant a^{-1}(b(s))}\left(\int_{s}^{t} k^{q}(a(t), x) w^{q}(x) d x\right)^{1 / q}\left(\int_{a(t)}^{b(s)} v^{-p^{\prime}}(x) d x\right)^{1 / p^{\prime}}, \\
A_{1}^{(i)}=\sup _{s \in I_{i}} \sup _{s \leqslant t \leqslant a^{-1}(b(s))}\left(\int_{s}^{t} w^{q}(x) d x\right)^{1 / q}\left(\int_{a(t)}^{b(s)} k^{p^{\prime}}(x, t) v^{-p^{\prime}}(x) d x\right)^{1 / p^{\prime}}, \\
B_{k, 1}^{(i)}=\left(\int_{a\left(\xi_{k}^{(i)}\right)}^{a\left(\xi_{k+1}^{(i)}\right)}\left(\int_{\xi_{k}^{(i)}}^{a^{-1}(t)} k^{q}(t, x) w^{q}(x) d x\right)^{r / q}\right. \\
\left.B_{k, 2}^{(i)}=\left(\int_{\xi_{k}^{(i)}}^{\xi_{k+1}^{(i)}}\left(\int_{\xi_{k}^{(i)}}^{t} w^{q}(x) d x\right)^{r\left(\xi_{k+1}^{(i)}\right)} v^{-p^{\prime}}(x) d x\right)^{r / q^{\prime}} v^{-p^{\prime}}(t) d t\right)^{1 / r}, \\
\left.\times\left(\int_{a(t)}^{a\left(\xi_{k+1}^{(i)}\right)} k^{p^{\prime}}(x, t) v^{-p^{\prime}}(x) d x\right)^{r / p^{\prime}} w^{q}(t) d t\right)^{1 / r},
\end{gathered}
$$


6

Hardy operator with variable limits on monotone functions

$$
\begin{aligned}
B_{k, 3}^{(i)}= & \left(\int_{b\left(\xi_{k}^{(i)}\right)}^{b\left(\xi_{k+1}^{(i)}\right)}\left(\int_{b^{-1}(t)}^{\xi_{k+1}^{(i)}} k^{q}(t, x) w^{q}(x) d x\right)^{r / q}\right. \\
& \left.\times\left(\int_{b\left(\xi_{k}^{(i)}\right)}^{t} v^{-p^{\prime}}(x) d x\right)^{r / q^{\prime}} v^{-p^{\prime}}(t) d t\right)^{1 / r}, \\
B_{k, 4}^{(i)}= & \left(\int_{\xi_{k}^{(i)}}^{\xi_{k+1}^{(i)}}\left(\int_{t}^{\xi_{k+1}^{(i)}} w^{q}(x) d x\right)^{r / p}\right. \\
& \left.\times\left(\int_{b\left(\xi_{k}^{(i)}\right)}^{b(t)} k^{p^{\prime}}(x, t) v^{-p^{\prime}}(x) d x\right)^{r / p^{\prime}} w^{q}(t) d t\right)^{1 / r},
\end{aligned}
$$

and the sequence $\left\{\xi_{k}^{(i)}\right\}_{k \in \mathbb{Z}_{i}}, \mathbb{Z}_{i} \subset \mathbb{Z}$ is such that $\xi_{0}^{(i)} \in I_{i}$ and $\xi_{k}^{(i)}=\left(a^{-1} \circ\right.$ $b)^{k}\left(\xi_{0}^{(i)}\right)$. Analogously, we can estimate the norm of the operator $K f(x)$ for $x \in J_{j}$. Since the kernel $k(y, x)$ satisfies the condition (9) and $b(x)<a(x)$, then

$$
\mathbb{A}_{j} \approx \begin{cases}A_{j}=A_{0}^{(j)}+A_{1}^{(j)}, & 1<p \leqslant q<\infty, \\ B_{j}=\left[\sum_{k \in \mathbb{Z}_{j}}\left(B_{k, 1}^{(j)}+B_{k, 2}^{(j)}+B_{k, 3}^{(j)}+B_{k, 4}^{(j)}\right)^{r}\right]^{1 / r}, & 1<q<p<\infty,\end{cases}
$$

where

$$
\begin{gathered}
A_{0}^{(j)}=\sup _{s \in J_{j}} \sup _{s \leqslant t \leqslant b^{-1}(a(s))}\left(\int_{s}^{t} k^{q}(b(t), x) w^{q}(x) d x\right)^{1 / q}\left(\int_{b(t)}^{a(s)} v^{-p^{\prime}}(x) d x\right)^{1 / p^{\prime}}, \\
A_{1}^{(j)}=\sup _{s \in J_{j}} \sup _{s \leqslant t \leqslant b^{-1}(a(s))}\left(\int_{s}^{t} w^{q}(x) d x\right)^{1 / q}\left(\int_{b(t)}^{a(s)} k^{p^{\prime}}(x, t) v^{-p^{\prime}}(x) d x\right)^{1 / p^{\prime}}, \\
B_{k, 1}^{(j)}=\left(\int_{b\left(\xi_{k}^{(j)}\right)}^{b\left(\xi_{k+1}^{(j)}\right)}\left(\int_{\xi_{k}^{(j)}}^{b^{-1}(t)} k^{q}(t, x) w^{q}(x) d x\right)^{r / q}\right. \\
\left.\times\left(\int_{t}^{b\left(\xi_{k+1}^{(j)}\right)} v^{-p^{\prime}}(x) d x\right)^{r / q^{\prime}} v^{-p^{\prime}}(t) d t\right)^{1 / r}
\end{gathered}
$$




$$
\begin{aligned}
& B_{k, 2}^{(j)}=\left(\int_{\xi_{k}^{(j)}}^{\xi_{k+1}^{(j)}}\left(\int_{\xi_{k}^{(j)}}^{t} w^{q}(x) d x\right)^{r / p}\right. \\
&\left.\times\left(\int_{b(t)}^{b\left(\xi_{k+1}^{(j)}\right)} k^{p^{\prime}}(x, t) v^{-p^{\prime}}(x) d x\right)^{r / p^{\prime}} w^{q}(t) d t\right)^{1 / r}, \\
& B_{k, 3}^{(j)}=\left(\int_{a\left(\xi_{k}^{(j)}\right)}^{a\left(\xi_{k+1}^{(j)}\right)}\left(\int_{a^{-1}(t)}^{\xi_{k+1}^{(j)}} k^{q}(t, x) w^{q}(x) d x\right)^{r / q}\right. \\
& \times\left.\left(\int_{a\left(\xi_{k}^{(j)}\right)}^{t} v^{-p^{\prime}}(x) d x\right)^{r / q^{\prime}} v^{-p^{\prime}}(t) d t\right)^{1 / r}, \\
& B_{k, 4}^{(j)}=\left(\int _ { \xi _ { k } ^ { ( j ) } } ^ { \xi _ { k + 1 } ^ { ( j ) } } \left(\int_{t}^{\xi_{k+1}^{(j)}} w^{q(x) d x)^{r / p}}\right.\right. \\
&\left.\times\left(\int_{a\left(\xi_{k}^{(j)}\right)}^{a(t)} k^{p^{\prime}}(x, t) v^{-p^{\prime}}(x) d x\right)^{r / p^{\prime}} w^{q}(t) d t\right)^{1 / r}
\end{aligned}
$$

and the sequence $\left\{\xi_{k}^{(j)}\right\}_{k \in \mathbb{Z}_{j}}, \mathbb{Z}_{j} \subset \mathbb{Z}$ is such as $\xi_{0}^{(j)} \in J_{j}$ and $\xi_{k}^{(j)}=\left(b^{-1} \circ\right.$ $a)^{k}\left(\xi_{0}^{(j)}\right)$.

On the base of the above estimates and Lemma 1 we obtain

Theorem 1. Let $1<p \leqslant q<\infty$, then the least constant $C$ of inequality (13) is equivalent to $\mathbb{A}$, where

$$
\mathbb{A}=\sup _{i \in \Sigma_{1}} A_{i}+\sup _{j \in \Sigma_{2}} A_{j}
$$

If $1<q<p<\infty$, then $C \approx \mathbb{B}$, where

$$
\mathbb{B}=\left(\sum_{i \in \Sigma_{1}} B_{i}^{r}\right)^{1 / r}+\left(\sum_{j \in \Sigma_{2}} B_{j}^{r}\right)^{1 / r}
$$

Remark 2. Theorem 1 has a counterpart for the operator

$$
\bar{K} f(x)=\int_{a(x)}^{b(x)} k(x, y) f(y) d y,
$$

where the kernel $k(x, y) \geqslant 0$ satisfies the conditions

$$
D^{-1} k(x, y) \leqslant k(x, b(z))+k(z, y) \leqslant D k(x, y)
$$


and

$$
D^{-1} k(x, y) \leqslant k(x, a(z))+k(z, y) \leqslant D k(x, y)
$$

instead of (8) and (9), respectively. We omit details which can be restored by using ([3], Theorem 3).

Theorem 1 has a particularly transparent form, when $k(y, x)=1$, with the help of the following

Definition 1. Given boundary functions $a(x)$ and $b(x)$, satisfying the conditions $(2)$, a number $p \in(1, \infty)$ and a weight function $v(x)$ such that $0<v(x)<\infty$ almost everywhere for $x \in \mathbb{R}^{+}, v^{p}(x)$ and $v^{-p^{\prime}}(x), p^{\prime}=$ $p /(p-1)$, are locally integrable on $\mathbb{R}^{+}$, we define fairway - the function $\sigma(x)$ such that $\min (a(x), b(x))<\sigma(x)<\max (a(x), b(x)), x \notin\left\{x_{k}\right\}$ and

$\int_{\min (a(x), b(x))}^{\sigma(x)} v^{-p^{\prime}}(y) d y=\int_{\sigma(x)}^{\max (a(x), b(x))} v^{-p^{\prime}}(y) d y \quad$ for all $x \in \mathbb{R}^{+} \backslash X$.

Put

$$
\begin{aligned}
& \Delta(x)=[\min (a(x), b(x)), \max (a(x), b(x))], \\
& \delta(x)= \begin{cases}{\left[b^{-1}(\sigma(x)), a^{-1}(\sigma(x))\right],} & x \in I, \\
{\left[a^{-1}(\sigma(x)), b^{-1}(\sigma(x))\right],} & x \in J,\end{cases}
\end{aligned}
$$

where $a^{-1}(y)$ and $b^{-1}(y)$ are the functions converse to $y=a(x)$ and $y=$ $b(x)$, respectively. Applying Theorem 1 of [3] we obtain the following

Theorem 2. Let the operator $\mathbb{H}$ of the form (1) be given with $u=1$ and the boundary functions $a(x)$ and $b(x)$ satisfying the conditions (2), (47). Then for the norm of $\mathbb{H}$ and $1<p \leqslant q<\infty$ the estimate

$$
\alpha_{0}(p, q) \mathcal{A} \leqslant\|\mathbb{H}\|_{L_{v}^{p} \rightarrow L_{w}^{q}} \leqslant \alpha_{1}(p, q) \mathcal{A},
$$

holds, where

$$
\begin{gathered}
\mathcal{A}=\sup _{t>0}\left(\int_{\delta(t)} w^{q}(y) d y\right)^{1 / q}\left(\int_{\Delta(t)} v^{-p^{\prime}}(y) d y\right)^{1 / p^{\prime}} . \\
\text { If } 0<q<p<\infty, p>1,1 / r=1 / q-1 / p, \text { then } \\
\beta_{0}(p, q) \mathcal{B} \leqslant\|\mathbb{H}\|_{L_{v}^{p} \rightarrow L_{w}^{q}} \leqslant \beta_{1}(p, q) \mathcal{B},
\end{gathered}
$$

where

$$
\mathcal{B}=\left(\int_{0}^{\infty}\left(\int_{\delta(t)} w^{q}(y) d y\right)^{r / p}\left(\int_{\Delta(t)} v^{-p^{\prime}}(y) d y\right)^{r / p^{\prime}} w^{q}(t) d t\right)^{1 / r}
$$




\section{Sawyer criterion}

Let

$$
T f(x)=\int_{0}^{\infty} t(x, y) f(y) d y
$$

be an integral operator with a non-negative kernel and

$$
T^{*} g(y)=\int_{0}^{\infty} t(x, y) g(x) d x
$$

is formally adjoint to $T$. If, for instance,

$$
H f(x)=\int_{0}^{x} f(y) d y
$$

then

$$
H^{*} g(y)=\int_{y}^{\infty} g(x) d x .
$$

By the Sawyer criterion [2] the weighted inequality

$$
\left(\int_{0}^{\infty}(T f)^{q}(x) w^{q}(x) d x\right)^{1 / q} \leqslant C\left(\int_{0}^{\infty} f^{p}(x) v^{p}(x) d x\right)^{1 / p}, \quad 0 \leqslant f \downarrow,
$$

for all non-increasing function $f$ is equivalent, when $1<p, q<\infty$, to the following two inequalities

$$
\left(\int_{0}^{\infty}\left(H T^{*} g\right)^{p^{\prime}}(x) V^{-p^{\prime}}(x) v^{p}(x) d x\right)^{1 / p^{\prime}} \leqslant C_{1}\left(\int_{0}^{\infty} g^{q^{\prime}}(x) w^{-q^{\prime}}(x) d x\right)^{1 / q^{\prime}}
$$

and

$$
\int_{0}^{\infty}\left(T^{*} g\right)(x) d x \leqslant C_{2}\left(\int_{0}^{\infty} v^{p}(x) d x\right)^{1 / p}\left(\int_{0}^{\infty} g^{q^{\prime}}(x) w^{-q^{\prime}}(x) d x\right)^{1 / q^{\prime}},
$$

where $g \geqslant 0, p^{\prime}=p /(p-1), q^{\prime}=q /(q-1)$ and $V(x)=\int_{0}^{x} v^{p}(y) d y$. We assume the constants $C, C_{1}$ and $C_{2}$ as the least possible. The second inequality (21) is easily characterized by the duality in Lebesgue's space and

$$
C_{2}=A_{0}:=\left(\int_{0}^{\infty}\left(\int_{0}^{\infty} t(x, y) d y\right)^{q} w^{q}(x) d x\right)^{1 / q}\left(\int_{0}^{\infty} v^{p}(x) d x\right)^{-1 / p} .
$$

As for the first inequality (20) it is more convenient for our purpose to use the dual form of $(20)$ 


$$
\left(\int_{0}^{\infty}\left(T H^{*} g\right)^{q}(x) w^{q}(x) d x\right)^{1 / q} \leqslant C_{1}\left(\int_{0}^{\infty} g^{p}(x) V^{p}(x) v^{-p^{2} / p^{\prime}}(x) d x\right)^{1 / p},
$$

for all $g \geqslant 0$.

Thus, if we characterize (23) by a finiteness of, say, a constant $A_{1}$, then for (19) we have

$$
C \approx A_{0}+A_{1}
$$

The similar criterion is valid for the inequality (19) restricted to the cone of non-decreasing functions. We omit details (see [8]).

\section{Main result}

Let $a(x)$ and $b(x)$ satisfy the assumptions of $\S 1$ and 2. Taking into account (6) and (7) we observe, that the inequality

$$
\left(\int_{0}^{\infty}|\mathbb{H} f(x)|^{q} w^{q}(x) d x\right)^{1 / q} \leqslant C\left(\int_{0}^{\infty} f^{p}(x) v^{p}(x) d x\right)^{1 / p}, \quad 0 \leqslant f \downarrow,
$$

is equivalent to

$$
\left(\int_{0}^{\infty}[\overline{\mathbb{H}} f(x)]^{q} w^{q}(x) d x\right)^{1 / q} \leqslant C\left(\int_{0}^{\infty} f^{p}(x) v^{p}(x) d x\right)^{1 / p}, \quad 0 \leqslant f \downarrow,
$$

where

$$
\overline{\mathbb{H}} f(x)= \begin{cases}\mathbb{H} f(x), & x \in I_{i}, \\ -\mathbb{H} f(x), & x \in J_{j}\end{cases}
$$

By the Sawyer criterion for the least possible constant $C$ in (26) we have

$$
C \approx A_{0}+A_{1},
$$

where

$$
A_{0}=\left(\int_{0}^{\infty}\left|\int_{a(x)}^{b(x)} u(y) d y\right|^{q} w^{q}(x) d x\right)^{1 / q}\left(\int_{0}^{\infty} v^{p}(x) d x\right)^{-1 / p}
$$

and $A_{1}$ is a characterization constant for the inequality

$$
\left(\int_{0}^{\infty}\left(\overline{\mathbb{H}} H^{*} g\right)^{q}(x) w^{q}(x) d x\right)^{1 / q} \leqslant C_{1}\left(\int_{0}^{\infty} g^{p}(x) V^{p}(x) v^{-p^{2} / p^{\prime}}(x) d x\right)^{1 / p}
$$


for all $g \geqslant 0$. Using $(27)$ and notations of $\S 2$ we write for the left hand side of $(29)$

$$
\begin{aligned}
\Phi:= & \int_{0}^{\infty}\left(\overline{\mathbb{H}} H^{*} g\right)^{q}(x) w^{q}(x) d x \\
= & \sum_{i \in \Sigma_{1}} \int_{t_{i}}^{t_{i+1}}\left(\int_{a(x)}^{b(x)} u(s) d s \int_{s}^{\infty} g(y) d y\right)^{q} w^{q}(x) d x \\
& \quad+\sum_{j \in \Sigma_{2}} \int_{s_{j}}^{s_{j+1}}\left(\int_{b(x)}^{a(x)} u(s) d s \int_{s}^{\infty} g(y) d y\right)^{q} w^{q}(x) d x \\
=: & \sum_{i \in \Sigma_{1}} F_{i}+\sum_{j \in \Sigma_{2}} G_{j} .
\end{aligned}
$$

Plainly

$$
\begin{aligned}
F_{i} \approx & \int_{t_{i}}^{t_{i+1}}\left(\int_{a(x)}^{b(x)} u(s) d s\right)^{q}\left(\int_{b(x)}^{\infty} g(y) d y\right)^{q} w^{q}(x) d x \\
& +\int_{t_{i}}^{t_{i+1}}\left(\int_{a(x)}^{b(x)}\left(\int_{a(x)}^{y} u(s) d s\right) g(y) d y\right)^{q} w^{q}(x) d x \\
G_{j} \approx & \int_{s_{j}}^{s_{j+1}}\left(\int_{b(x)}^{a(x)} u(s) d s\right)^{q}\left(\int_{a(x)}^{\infty} g(y) d y\right)^{q} w^{q}(x) d x \\
& +\int_{s_{j}}^{s_{j+1}}\left(\int_{b(x)}^{a(x)}\left(\int_{b(x)}^{y} u(s) d s\right) g(y) d y\right)^{q} w^{q}(x) d x,
\end{aligned}
$$

Hence,

$$
\begin{aligned}
\Phi \approx & \int_{0}^{\infty}\left|\int_{a(x)}^{b(x)} u(s) d s\right|^{q}\left(\int_{\max (a(x), b(x))}^{\infty} g(y) d y\right)^{q} w^{q}(x) d x \\
& +\int_{0}^{\infty}\left|\int_{a(x)}^{b(x)} \mathbf{k}(y, x) g(y) d y\right|^{q} w^{q}(x) d x,
\end{aligned}
$$

where

$$
\mathbf{k}(y, x):= \begin{cases}\int_{a(x)}^{y} u(s) d s, & a(x) \leqslant y \leqslant b(x), x \in I_{i}, \\ \int_{b(x)}^{y} u(s) d s, & b(x) \leqslant y \leqslant a(x), x \in J_{j} .\end{cases}
$$

Clearly, the kernel $\mathbf{k}(y, x) \geqslant 0$ satisfies the condition (8) and (9) with $D=1$. Thus, the inequality (29) is equivalent to the following two inequalities 
$\begin{aligned}(31)\left(\int_{0}^{\infty}(\right. & \left.\left.\int_{\max (a(x), b(x))}^{\infty} g(y) d y\right)^{q}\left|\int_{a(x)}^{b(x)} u(s) d s\right|^{q} w^{q}(x) d x\right)^{1 / q} \\ & \leqslant C_{1,0}\left(\int_{0}^{\infty} g^{p}(x) V^{p}(x) v^{-p^{2} / p^{\prime}}(x) d x\right)^{1 / p}, \quad g \geqslant 0,\end{aligned}$

and

$$
\begin{aligned}
(32)\left(\int_{0}^{\infty} \mid\right. & \left.\left.\int_{a(x)}^{b(x)} \mathbf{k}(y, x) g(y) d y\right|^{q} w^{q}(x) d x\right)^{1 / q} \\
& \leqslant C_{1,1}\left(\int_{0}^{\infty} g^{p}(x) V^{p}(x) v^{-p^{2} / p^{\prime}}(x) d x\right)^{1 / p}, \quad g \geqslant 0,
\end{aligned}
$$

with $C_{1} \approx C_{1,0}+C_{1,1}$. Characterization of (31) is known, because $\max (a(x), b(x))$ is an increasing function, $C_{1,0} \approx A_{1,0}$, where for $1<p \leqslant$ $q<\infty$

$$
\text { (33) } \begin{aligned}
A_{1,0}:= & \sup _{t>0}\left(\int_{0}^{t}\left|\int_{a(x)}^{b(x)} u(s) d s\right|^{q} w^{q}(x) d x\right)^{1 / q} \\
& \times\left(\int_{\max (a(t), b(t))}^{\infty} V^{-p^{\prime}}(x) v^{p}(x) d x\right)^{1 / p^{\prime}},
\end{aligned}
$$

and for $1<q<p<\infty$ with $1 / r=1 / q-1 / p$

$$
\text { (34) } \begin{aligned}
A_{1,0}:= & \left(\int_{0}^{\infty}\left(\int_{0}^{t}\left|\int_{a(x)}^{b(x)} u(s) d s\right|^{q} w^{q}(x) d x\right)^{r / p}\right. \\
& \left.\times\left(\int_{\max (a(t), b(t)}^{\infty} V^{-p^{\prime}}(x) v^{p}(x) d x\right)^{r / p^{\prime}}\left|\int_{a(t)}^{b(t)} u(s) d s\right|^{q} w^{q}(t) d t\right)^{1 / r}
\end{aligned}
$$

Applying Theorem 1 we obtain the main result of the paper.

Theorem 3. For the least possible constant in the inequality (25) the two-sided estimate

holds,

$$
C \approx A_{0}+A_{1,0}+A_{1,1}
$$

where $A_{0}$ and $A_{1,0}$ are given by (28) and (33) - (34), respectively, and

$$
\begin{aligned}
(35) A_{1,1}= & \sup _{i \in \Sigma_{1}}\left[\sup _{s \in I_{i}} \sup _{s \leqslant t \leqslant a^{-1}(b(s))}\left(A_{0}(s, t)+A_{1}(s, t)\right)\right] \\
& +\sup _{j \in \Sigma_{2}}\left[\sup _{s \in J_{j}} \sup _{s \leqslant t \leqslant b^{-1}(a(s))}\left(A_{0}(s, t)+A_{1}(s, t)\right)\right], 1<p \leqslant q<\infty,
\end{aligned}
$$




$$
\text { (36) } \begin{aligned}
A_{1,1}= & \left(\sum_{i \in \Sigma_{1}} \sum_{k \in \mathbb{Z}_{i}} B_{k, 1}^{r}+B_{k, 2}^{r}+B_{k, 3}^{r}+B_{k, 4}^{r}\right)^{1 / r} \\
& +\left(\sum_{j \in \Sigma_{2}} \sum_{k \in \mathbb{Z}_{j}} B_{k, 1}^{r}+B_{k, 2}^{r}+B_{k, 3}^{r}+B_{k, 4}^{r}\right)^{1 / r}, 1<q<p<\infty,
\end{aligned}
$$

$$
\begin{aligned}
A_{0}(s, t)= & \left(\int_{s}^{t} \mathbf{k}^{q}(\min (a(t), b(t)), x) w^{q}(x) d x\right)^{1 / q} \\
& \times\left(\int_{\min (a(t), b(t))}^{\max (a(s), b(s))} V^{-p^{\prime}}(x) v^{p}(x) d x\right)^{1 / p^{\prime}}, \\
A_{1}(s, t)= & \left(\int_{s}^{t} w^{q}(x) d x\right)^{1 / q} \\
& \times\left(\int_{\min (a(t), b(t))}^{\max (a(s), b(s))} \mathbf{k}^{p^{\prime}}(x, t) V^{-p^{\prime}}(x) v^{p}(x) d x\right)^{1 / p^{\prime}}, \\
B_{k, 1}= & \left(\int_{\min \left(a\left(\xi_{k}\right), b\left(\xi_{k}\right)\right)}^{\min \left(a\left(\xi_{k+1}\right), b\left(\xi_{k+1}\right)\right)}\left(\int_{\xi_{k}}^{\max \left(a^{-1}(t), b^{-1}(t)\right)} \mathbf{k}^{q}(t, x) w^{q}(x) d x\right)^{r / q}\right. \\
& \left.\times\left(\int_{t}^{\min \left(a\left(\xi_{k+1}\right), b\left(\xi_{k+1}\right)\right)} V^{-p^{\prime}}(x) v^{p}(x) d x\right)^{r / q^{\prime}} V^{-p^{\prime}}(t) v^{p}(t) d t\right)^{1 / r}, \\
& \left.\left.\times\left(\int_{\max \left(a\left(\xi_{k}\right), b\left(\xi_{k}\right)\right)}^{t} V^{-p^{\prime}}(x) v^{p}(x) d x\right)^{r / q^{\prime}} V^{-p^{\prime}}(t) v^{p}(t) d t\right)^{1 / r}, \mathbf{k}^{q}(t, x) w^{q}(x) d x\right)^{r / q} \\
B_{k, 2}= & \left(\int_{\xi_{k}}^{\xi_{k+1}}\left(\int_{\xi_{k}}^{t} w^{q}(x) d x\right)^{r / p}\right. \\
B_{k, 3}= & \times\left(\int_{\max \left(a\left(\xi_{k}\right), b\left(\xi_{k}\right)\right)}^{\max \left(a\left(\xi_{k+1}\right), b\left(\xi_{k+1}\right)\right)}\left(\int_{\min \left(a^{-1}(t), b^{-1}(t)\right)}^{\min \left(a\left(\xi_{k+1}\right), b\left(\xi_{k+1}\right)\right)} \mathbf{k}^{p^{\prime}}(x, t) V^{-p^{\prime}}(x) v^{p}(x) d x\right)^{r / p^{\prime}} w^{q}(t) d t\right)^{1 / r},
\end{aligned}
$$




$$
\begin{aligned}
B_{k, 4}= & \left(\int_{\xi_{k}}^{\xi_{k+1}}\left(\int_{t}^{\xi_{k+1}} w^{q}(x) d x\right)^{r / p}\right. \\
& \left.\times\left(\int_{\max \left(a\left(\xi_{k}\right), b\left(\xi_{k}\right)\right)}^{\max (a(t), b(t))} \mathbf{k}^{p^{\prime}}(x, t) V^{-p^{\prime}}(x) v^{p}(x) d x\right)^{r / p^{\prime}} w^{q}(t) d t\right)^{1 / r},
\end{aligned}
$$

and

$$
\xi_{k}=\left\{\begin{array}{lll}
\left(a^{-1} \circ b\right)^{k}\left(\xi_{0}\right), & \xi_{0} \in I_{i}, & \text { if } a(x)<b(x), \\
\left(b^{-1} \circ a\right)^{k}\left(\xi_{0}\right), & \xi_{0} \in J_{j}, & \text { if } b(x)<a(x) .
\end{array}\right.
$$

Remark 3. The similar result as Theorem 3 is also true for non-decreasing functions. We omit details.

Acknowledgements. The paper was composed during the visit of the second author to Department of Mathematics of Korean Advanced Institute of Science and Technology. The author thanks the Institute for hospitality and support. Also the authors thank Dmitry V. Prokhorov for discussion.

\section{References}

[1] H.P. Heinig and G. Sinnamon, Mapping properties of integral averaging operators, Studia Math., 129 (1998), 157-177.

[2] E.T. Sawyer, Boundedness of classical operators on classical Lorents spaces, Studia Math., 96 (1990), 145-158.

[3] V.D. Stepanov and E.P. Ushakova, On integral operators with variable limits of integration, Proceedings of the Steklov Institute of Mathematics, 232 (2001), 298-317.

[4] T. Chen and G. Sinnamon, Generalized Hardy operators and normalizing measures, J. Ineq. Appl., 7 (2002), 829-866.

[5] A. Gogatishvili and J. Lang, The generalized Hardy operators with kernel and variable integral limits in Banach functions spaces, J. Ineq. Appl., 4 (1999), 1-16.

[6] W. Rudin, Functional Analysis, 2nd edition, McGraw-Hill, New York, 1991.

[7] D.V. Prokhorov and V.D. Stepanov, Weighted estimates of RiemannLiouville operators and applications, Proceedings of the Steklov Institute of Mathematics, 2003, to appear.

[8] V.D. Stepanov, Integral operators on the cone of monotone functions, J. London Math. Soc., (2) 48 (1993), 465-487. 
Department of Mathematics

KAIST, 373-1 Kusong-Dong

Yusong-Gu, Taejon, 305-701

SOUTH KOREA

(Email : vstep@math.kaist.ac.kr)

Computing Centre FEB RAS

Tikhookeanskaya 153

Khabarovsk, 680042

RUSSIA

(Email : ushakova@as.khb.ru)

(Received : February 2003) 


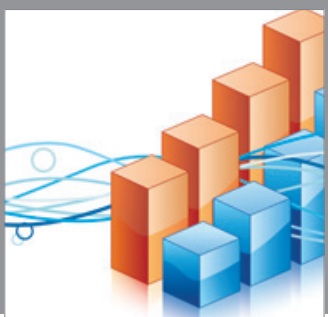

Advances in

Operations Research

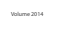

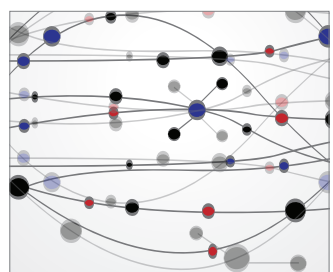

\section{The Scientific} World Journal
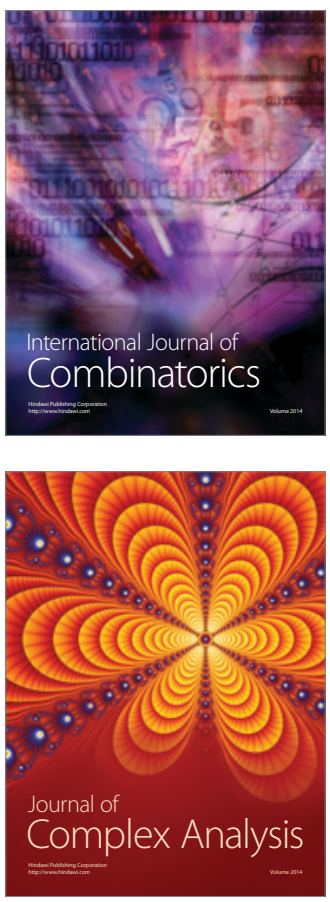

International Journal of

Mathematics and

Mathematical

Sciences
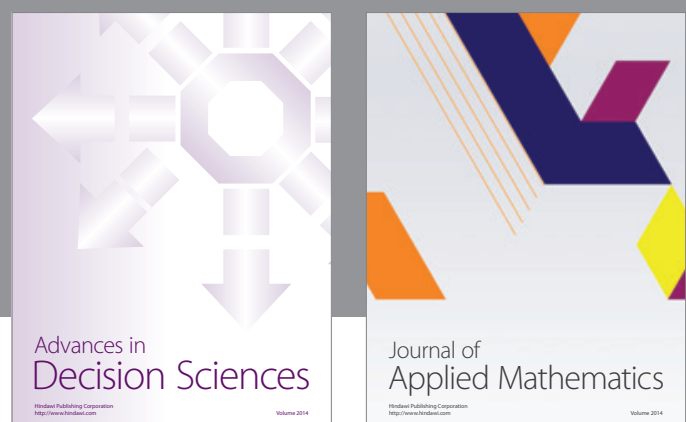

Journal of

Applied Mathematics
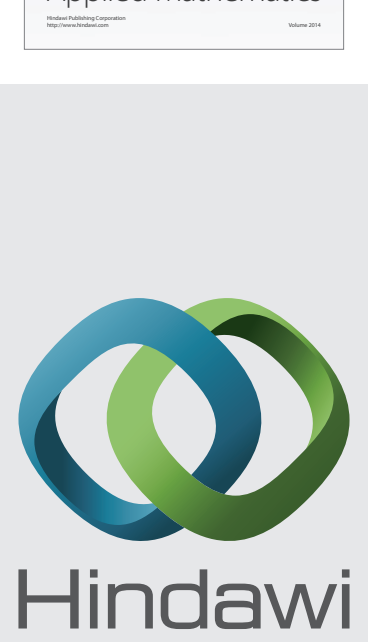

Submit your manuscripts at http://www.hindawi.com
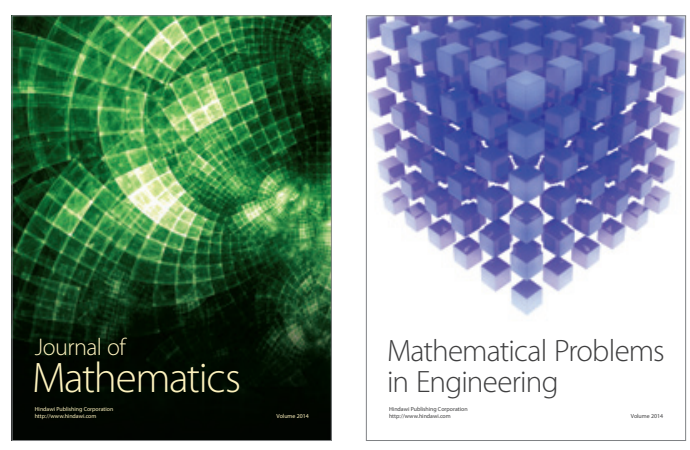

Mathematical Problems in Engineering
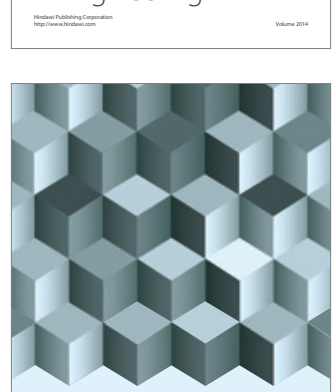

Journal of

Function Spaces
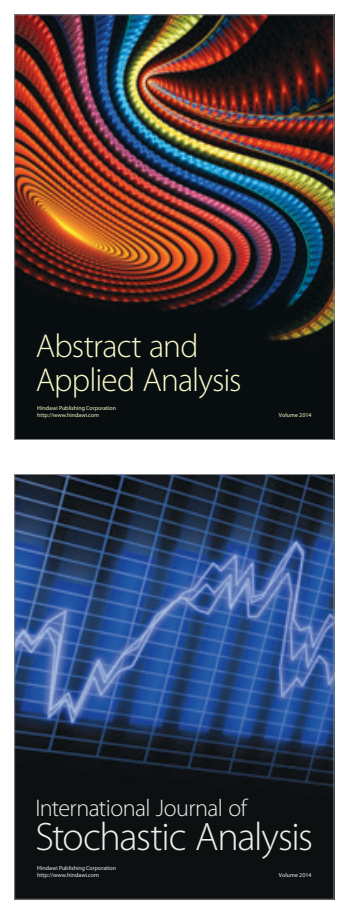

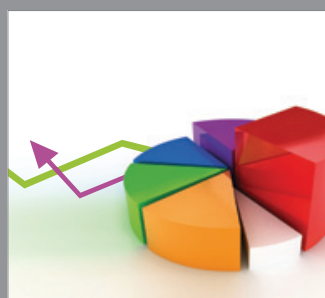

ournal of

Probability and Statistics

Promensencen
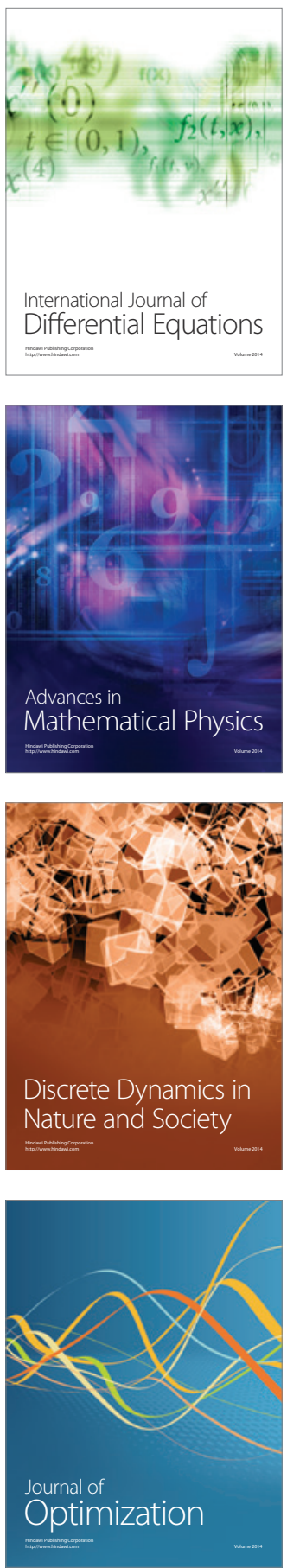